LBNL-52581

\title{
A MODEL OF ATL GROUND MOTION FOR STORAGE RINGS
}

\author{
A. Wolski \\ Ernest Orlando Lawrence Berkeley National Laboratory, Berkeley, CA 94720 \\ N.J. Walker \\ DESY, Hamburg, Germany \\ Presented at PAC 2003, Portland, Oregon, May 12-16, 2003
}

\begin{abstract}
Low emittance electron storage rings, such as those used in third generation light sources or linear collider damping rings, rely for their performance on highly stable alignment of the lattice components. Even if all vibration and environmental noise sources could be suppressed, diffusive ground motion will lead to orbit drift and emittance growth. Understanding such motion is important for predicting the performance of a planned accelerator and designing a correction system. A description (known as the ATL model) of ground motion over relatively long time scales has been developed and has become the standard for studies of the long straight beamlines in linear colliders. Here, we show how the model may be developed to include beamlines of any geometry. We apply the model to the NLC and TESLA damping rings, to compare their relative stability under different conditions.
\end{abstract}

\section{Disclaimer}

This document was prepared as an account of work sponsored by the United States Government. While this document is believed to contain correct information, neither the United States Government nor any agency thereof, nor The Regents of the University of California, nor any of their employees, makes any warranty, express or implied, or assumes any legal responsibility for the accuracy, completeness, or usefulness of any information, apparatus, product, or process disclosed, or represents that its use would not infringe privately owned rights. Reference herein to any specific commercial product, process, or service by its trade name, trademark, manufacturer, or otherwise, does not necessarily constitute or imply its endorsement, recommendation, or favoring by the United States Government or any agency thereof, or The Regents of the University of California. The views and opinions of authors expressed herein do not necessarily state or reflect those of the United States Government or any agency thereof or The Regents of the University of California.

LBNL is an equal opportunities employer.

This work was supported by the Director, Office of Science, of the U.S. Department of Energy under Contract No. DE-AC03-76SF00098. 


\title{
A MODEL OF ATL GROUND MOTION FOR STORAGE RINGS*
}

\author{
A. Wolski", LBNL, Berkeley, CA 94720, USA \\ N.J. Walker, DESY, Hamburg, Germany
}

\begin{abstract}
Low emittance electron storage rings, such as those used in third generation light sources or linear collider damping rings, rely for their performance on highly stable alignment of the lattice components. Even if all vibration and environmental noise sources could be suppressed, diffusive ground motion will lead to orbit drift and emittance growth. Understanding such motion is important for predicting the performance of a planned accelerator and designing a correction system. A description (known as the ATL model) of ground motion over relatively long time scales has been developed and has become the standard for studies of the long straight beamlines in linear colliders. Here, we show how the model may be developed to include beamlines of any geometry. We apply the model to the NLC and TESLA damping rings, to compare their relative stability under different conditions.
\end{abstract}

\section{ATL FOR LINEAR GEOMETRIES}

Damping rings for future linear colliders will need to operate with equilibrium vertical emittances of a few picometers (unnormalized). Achieving such highly focused beams will depend on precise alignment of beamline components, particularly the quadrupole and sextupole magnets, and effective steering and coupling correction algorithms. Small movements of the quadrupoles and sextupoles over time will necessitate (possibly invasive) retuning of the ring, and it is important to estimate at the design stage how frequently such tuning may be required. Although movement of magnets and diagnostic components can occur from a variety of causes, for example thermal effects or mechanical stress on the supports, a significant contribution to the motion is expected to come from the ground itself.

Ground motion in a variety of regimes relevant for high energy beamlines has been discussed in some detail by Napoli and Seryi [1]. In this paper, we consider only the slow or diffusive ground motion, which is described by the ATL model:

$$
\left\langle\left(Y_{i}-Y_{j}\right)^{2}\right\rangle=A T L_{i j}
$$

Here, $Y_{i}$ is the vertical position of the $i^{\text {th }}$ beamline component relative to some (conceptual) fixed reference point, $T$ is the time after initial (perfect) alignment, and $L_{i j}$ is the horizontal distance between components $i$ and $j$. The parameter $A$ is a constant characteristic of the site on which the accelerator is built: "quieter" or "more stable"

*Work supported by the US DOE, contract DE-AC03-76SF00098. \#awolski@lbl.gov sites have lower values of $A$. The angled brackets indicate an average over an ensemble, or in practice, the mean value over a number of different random seeds. In the original paper [1], the authors considered only the application of this model to linear systems, such as the main linac or beam delivery system of a linear collider. In such a system, it is straightforward to apply the ATL model (1) in a simulation. For a given time step $T$ it is only necessary to start at one end, giving each component a random displacement with respect to the previous component, with the variance of the displacement given by $A T L$, with $L$ the distance between the components. This generates a "random walk" and one finds that the relative displacement of any two components in the beamline then obeys the ATL model.

Attempting to apply this procedure to a storage ring will result in failure, since $L_{i j}$ becomes the arc length between two components, rather than the direct horizontal distance that is required by the model. The start and end points, for example, will typically be close together, but will have a relative misalignment characteristic of the circumference of the ring, which may be very large.

Since the ATL model is now the standard description for linacs and beam delivery systems in linear colliders [2], it is desirable to be able to apply the same model to describe the motion of components in other parts of the machine, in particular the damping rings, where alignment is an important issue. We therefore describe here a technique for applying the ATL model in a consistent way to beamlines that are not rectilinear.

\section{ATL FOR GENERAL PLANAR GEOMETRIES}

\section{Theoretical Analysis}

First, we choose a fixed reference point $j=0$. For any component $i$ we can then write:

$$
\left\langle Y_{i}^{2}\right\rangle=A T L_{i 0}
$$

Noting that:

$$
\left\langle\left(Y_{i}-Y_{j}\right)^{2}\right\rangle=\left\langle Y_{i}^{2}\right\rangle+\left\langle Y_{j}^{2}\right\rangle-2\left\langle Y_{i} Y_{j}\right\rangle
$$

it then follows that:

$$
\left\langle Y_{i} Y_{j}\right\rangle=A T \cdot \frac{1}{2}\left(L_{i 0}+L_{j 0}-L_{i j}\right)=A T M_{i j}
$$

which defines the matrix $\mathbf{M}$ with components $M_{i j}$. We can write equation (2) in terms of the outer product of the vector $\mathbf{Y}$ (with components $Y_{i}$ ) with itself:

$$
\left\langle\mathbf{Y} \cdot \mathbf{Y}^{\mathrm{T}}\right\rangle=A T \mathbf{M}
$$


Since $\mathbf{M}$ is symmetric, it can be diagonalized by a unitary matrix $\Lambda$ :

$$
\tilde{\mathbf{M}}=\boldsymbol{\Lambda} \cdot \mathbf{M} \cdot \boldsymbol{\Lambda}^{\mathrm{T}}
$$

The components of $\tilde{\mathbf{M}}$ are the eigenvalues $\lambda_{i}$ of $\mathbf{M}$ :

$$
\tilde{M}_{i j}=\lambda_{i} \delta_{i j}
$$

$\Lambda$ is of course constructed from the eigenvectors of $\mathbf{M}$. It is important that we choose an orthonormal basis for the eigenvectors, otherwise $\tilde{\mathbf{M}}$ is not diagonal - this is discussed further below. We define the vector $\mathbf{V}$ :

$$
\mathbf{V}=\boldsymbol{\Lambda} \cdot \mathbf{Y}
$$

By premultiplying equation (3) by $\Lambda$ and postmultiplying by $\Lambda^{\mathrm{T}}$, we see that the components of $\mathbf{V}$ satisfy:

$$
\left\langle V_{i}^{2}\right\rangle=A T \lambda_{i}
$$

Equation (4) is our result: it is all we need to apply the ATL ground motion model to a general planar geometry. Explicitly, from the distances between the components and a chosen fixed reference point, we construct the symmetric matrix $\mathbf{M}$, and find its eigensystem. We then generate a set of values with variances given by the eigenvalues of $\mathbf{M}$, and transform these values using the eigenvectors of $\mathbf{M}$ to find the corresponding vertical displacements of the components. The case of a rectilinear beamline is of course a special case of this general model.

\section{Implementation Issues}

We commented above that it is important to choose an orthonormal basis for the eigenvectors of $\mathbf{M}$. It is easy to show that if the eigenvalues are all distinct, then the eigenvectors of a symmetric matrix are necessarily orthogonal. However, eigenvectors corresponding to repeated eigenvalues may become "mixed" in such a way that they are not orthogonal. In this case, the matrix $\tilde{\mathbf{M}}$ is not diagonal, and the above procedure will fail, since the final result (4) is not correct.

The question then arises, as to which situations will give rise to repeated eigenvalues of $\mathbf{M}$ ? This is a difficult question to answer completely, but in general, repeated eigenvalues are an indication of some symmetry in the system. For example, one finds that if the system consists of $N$ points equally distributed on a circle with the reference point at the center, the eigenvalues occur in $(\mathrm{N}$ $2) / 2$ or $(N-1) / 2$ pairs according to whether $N$ is even or odd. Moving the reference point away from the center reduces the number of pairs of eigenvalues, and choosing a reference point on the circle renders them all distinct.

Fortunately, where repeated eigenvalues do occur, the above technique can still be applied: it is, however, necessary to ensure that the eigenvectors form and orthonormal basis.

Large accelerators (for example, the TESLA Damping Ring) may consist of many hundreds of components, in which case we need to find the eigensystem of a large matrix. This is not necessarily an obstacle, since efficient numerical algorithms exist for this task. Also, for a given accelerator design, it is only necessary to perform the eigensystem analysis once. The eigenvalues and eigenvectors can then be saved for application of the ATL model for simulations over different timescales.

\section{EXAMPLES}

\section{Regular Grid}

To test the technique, it is interesting to apply it to a regular grid of points. In this case, one expects to see the correlation of vertical displacement with distance to show up clearly (or not, in the case of some flaw in the theory or its application). The application is conveniently carried out in any mathematical program capable of an eigensystem analysis of a large matrix. We have used Mathematica [3]: our grid consisted of $25 \times 25$ points, with the reference point at the origin. Initially, all points are at $Y=0$. Figure 1 shows that grid after 5, 10, 30 and 50 time steps (the value of $A$ is $5 \times 10^{-4}$ ).

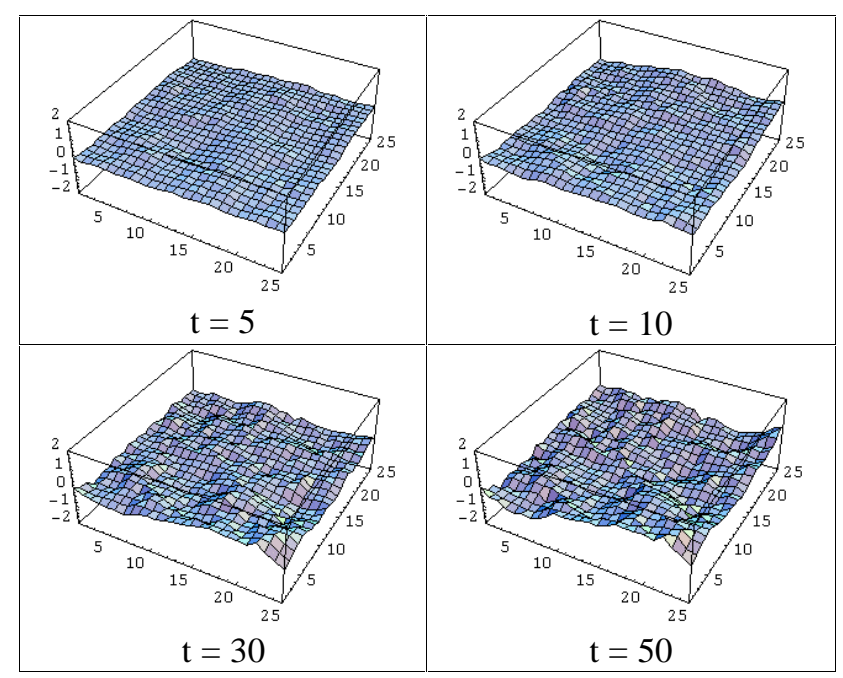

Figure 1: ATL Applied to a Rectangular Grid of Points

Clearly, the displacements of the points on the grid are correlated, with the average relative displacement between two points increasing with the distance between the points.

\section{The NLC Main Damping Rings}

For a storage ring, we are generally interested in questions such as the change in the closed orbit and the vertical emittance degradation when the main magnets in the lattice move. It is therefore convenient to have the ATL model integrated into an accelerator simulation code. We have implemented the 2D ATL model into the tracking code MERLIN [4], and applied the model to the NLC Main Damping Rings [5] and the TESLA Damping Rings [6].

Figure 2 shows the effects of ATL ground motion applied to the quadrupoles of the NLC Main Damping Ring over a period of 30 hours, starting from a perfect 
alignment. The blue points show the quadrupoles, and the red line shows the closed orbit. The value of $A$ used in the simulation was $4 \times 10^{-6} \mu \mathrm{m}^{2} / \mathrm{m} / \mathrm{s}$. The horizontal scales are in meters, and the vertical scales are in microns.

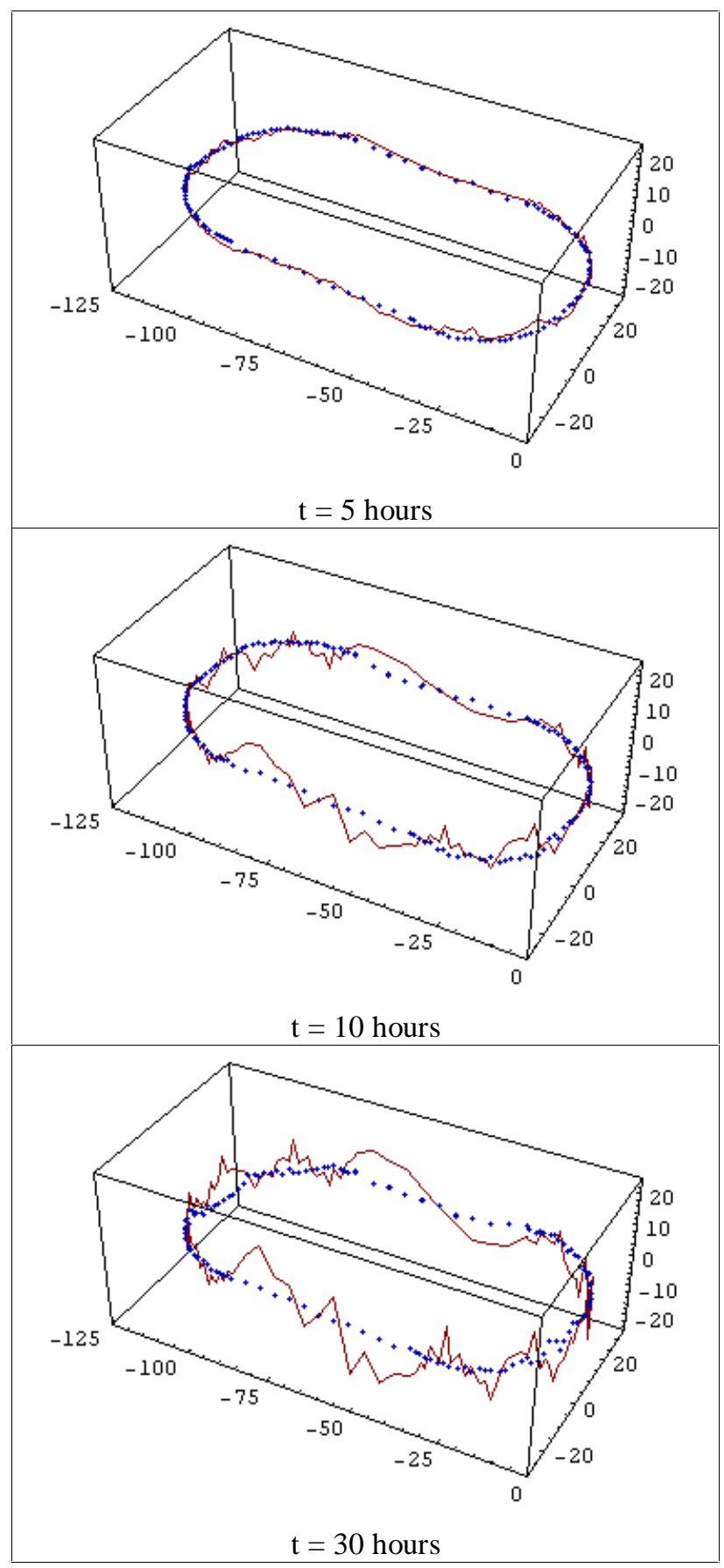

Figure 2: Effect of ATL Ground Motion on the Closed Orbit in the NLC Main Damping Rings

\section{EMITTANCE TUNING}

Maintenance of very low vertical emittance over long periods is an important issue for linear collider damping rings, as this has a direct impact on the rate at which the linear collider can integrate luminosity. Although it is expected that low emittance may be maintained to some extent by a straightforward orbit correction, it is important to know how frequently more invasive tuning procedures may be required.

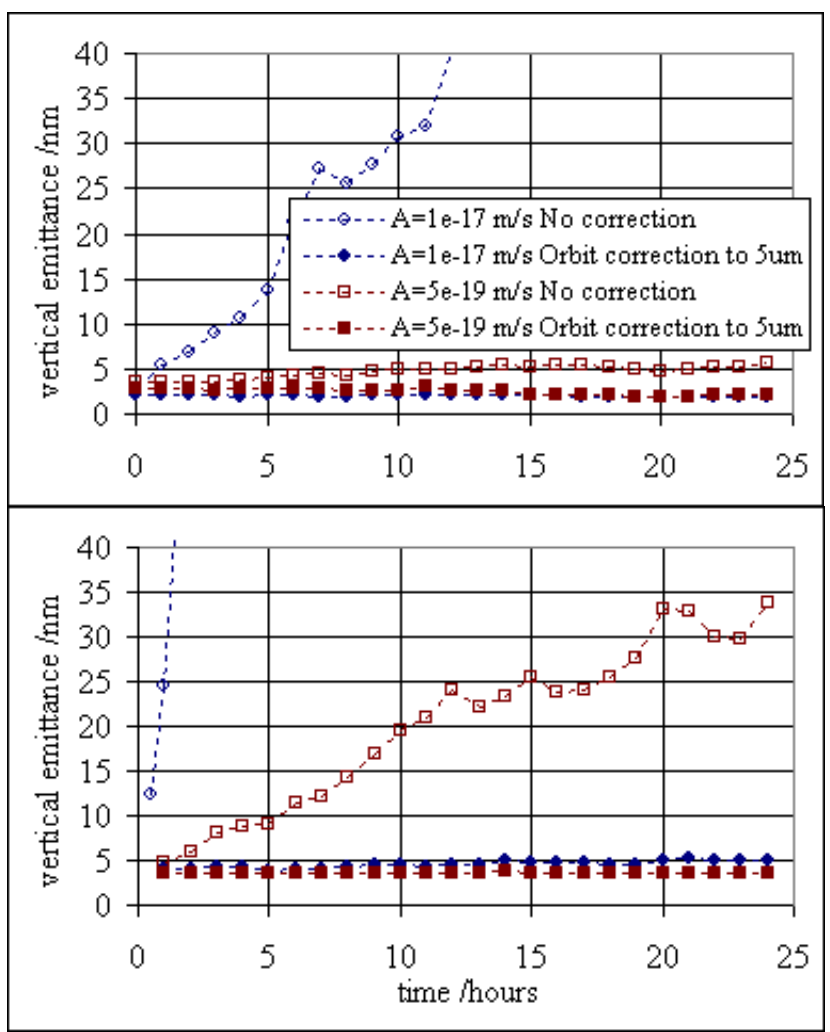

Figure 3: Emittance Growth from ATL Ground Motion in the NLC (top) and TESLA Damping Rings

Figure 3 shows the growth of the normalized vertical emittance from ATL ground motion in the NLC Main Damping Rings and the TESLA Damping Rings. The rings were initially misaligned by applying 50 hours of ATL ground motion to the quadrupoles and sextupoles, followed by a full coupling correction. Without orbit correction, a rapid emittance growth can be seen for $A=$ $10^{-17} \mathrm{~m} / \mathrm{s}$ (NLC and TESLA) and $A=5 \times 10^{-19} \mathrm{~m} / \mathrm{s}$ (TESLA). An orbit correction at half-hourly intervals to 5 $\mu \mathrm{m}$ is effective at suppressing the emittance growth.

\section{REFERENCES}

[1] A. Seryi and O. Napoly, "Influence of Ground Motion on the Time Evolution of Beams in Linear Colliders", Phys. Rev. E, 53 p.5323 (1996).

[2] Second Report of the International Linear Collider Technical Review Committee, 2003.

[3] Wolfram Research, http://www.wolfram.com/

[4] http://www.desy.de/ njwalker/MerlinII/index.htm

[5] A. Wolski and J. Corlett, "The Next Linear Collider Damping Ring Lattices", PAC'01, p.3795.

[6] TESLA Technical Design Report, Part II p.137, DESY 2001-011, March 2001. 